\title{
Gliomatosis cerebri: a monocentric real-life experience
}

\author{
Luisa Bellu ${ }^{1}$, Mario Caccese ${ }^{2}$, Giulia Cerretti ${ }^{2}$, Franco Berti ${ }^{1}$, Fabio Busato ${ }^{1}$, Alessandro Parisi ${ }^{1}$, Marta \\ Padovan $^{2}$, Vittorina Zagonel ${ }^{2}$, Giuseppe Lombardi ${ }^{2}$ \\ ${ }^{1}$ Radiotherapy Unit - Imaging and Medical Physics Department, Veneto Institute of Oncology - IOV - IRCCS, Padova 6435128, \\ Italy. \\ ${ }^{2}$ Department of Oncology, Oncology 1, Veneto Institute of Oncology - IOV - IRCCS, Padova 6435128, Italy.
}

Correspondence to: Dr. Luisa Bellu, Radiotherapy Unit - Imaging and Medical Physics Department, Veneto Institute of Oncology - IOV - IRCCS, Via Gattamelata, Padova 6435128, Italy. E-mail: luisa.bellu@iov.veneto.it

How to cite this article: Bellu L, Caccese M, Cerretti G, Berti F, Busato F, Parisi A, Padovan M, Zagonel V, Lombardi G. Gliomatosis cerebri: a monocentric real-life experience. J Cancer Metastasis Treat 2021;7:29.

https://dx.doi.org/10.20517/2394-4722.2021.89

Received: 2 Apr 2021 First Decision: 10 May 2021 Revised: 10 May 2021 Accepted: 19 May 2021 Published: 30 May 2021

Academic Editors: Lucio Miele, Yaoqi Alan Wang Copy Editor: Xi-Jun Chen Production Editor: Xi-Jun Chen

\begin{abstract}
Aim: Gliomatosis cerebri (GC) is defined as a rare pattern of growth of diffuse gliomas involving three or more cerebral lobes. Given its rarity, it is difficult to define prognostic factors and standard of treatment. We retrospectively analyzed patients (PT) with GC from a single institution with the aim of identifying the main prognostic factors and to assess optimal management.
\end{abstract}

Methods: Medical records were reviewed of patients $\geq 18$ years with a histological and/or radiological diagnosis of GC (with no contrast enhancement) occurring between 2006 and 2017. Median progression free survival (PFS) and overall survival (OS) were calculated by the Kaplan-Meier method.

Results: We analyzed 33 PT, 22 males and 11 females; Eastern Cooperative Oncology Group (ECOG) performance status (PS) was 0-1 in 21 of the patients. Twenty-two PT underwent biopsy: 16 were astrocytomas and 6 oligodendrogliomas. 06-methylguanin-DNA-methyltransferase (MGMT) was detected in 14 cases, and it was methylated in eight cases. Isocitrate dehydrogenase 1 (IDH1) was analyzed in 16 PT, and it had mutated in 10 of them. Nine PT (27\%) were treated with radiation therapy (RT) plus concurrent temozolomide (TMZ), 22 PT (67\%) received TMZ alone, and 2 PT (6\%) underwent RT alone. We reported "complete response" in 1 patient (3\%), partial response in 9 PT (27\%), and stable disease in 15 PT (45\%), while 8 PT (25\%) had a progressive 
disease. For all PT, PFS and OS were 19.1 and 30.7 months, respectively. For ECOG PS 0-1 and $\geq 2$, PFS was 34.6 months vs. 3.4 months $(P<0.0001)$ and OS was 42 months vs. 8.9 months $(P<0.0001)$, respectively. Methylated MGMT was associated with longer PFS (41.6 months vs. 8.9 months, $P=0.05$ ) and OS (52.7 months vs. 14.6 months, $P=0.009) ;$ PFS for IDH1 mutation and IDH wild-type was 52.7 months vs. 8.9 months $(P=0.006)$ and OS was 52.7 months vs. 41.7 months $(P=0.02)$, respectively. No significant difference was detected as regards treatments. With regard to histological subtype, OS was 42.0 months vs. 52.7 months $(P=0.8)$ and PFS was 41.6 months vs. 28.6 months ( $P=0.7$ ) for astrocytoma vs. oligodendroglioma, respectively. PT with treatment response showed a longer OS. PT receiving second-line treatment had a longer OS of 30.7 months vs. 6.5 months $(P=0.04)$.

Conclusion: ECOG PS, MGMT methylation, and IDH1 mutational status seem to have an important prognostic significance, while the type of treatment does not seem to affect survival. Treatment response could be a surrogate marker for survival.

Keywords: Low-grade gliomas, gliomatosis cerebri, temozolomide, IDH, radiotherapy

\section{INTRODUCTION}

According to the 2016 World Health Organization (WHO) Classification of tumors of the Central Nervous System, gliomatosis cerebri is no longer a distinct pathological entity, being designated as a pattern of diffuse and extensive growth of glioma cells. It includes astrocytic and oligodendroglial tumors, involving three or more cerebral lobes, usually bilateral, with a frequent extension to infratentorial structures ${ }^{[1]}$.

However, GC remains a complicated entity to treat, with great heterogeneity in clinical features and outcome. Symptoms and radiological appearance are non-specific, and, thus, it can be confused with other neurological diseases ${ }^{[2]}$; on magnetic resonance imaging (MRI), GC usually shows diffuse infiltration predominantly of white matter, with T2-weighted and fluid-attenuated inversion recovery (FLAIR) hyperintensity.

With regard to the treatment of GC, optimal management is still unclear. Surgery is used for focal biopsy and the role of radiotherapy and chemotherapy is discordant.

The aim of our paper is to describe our real-life experience in GC by retrospective analysis and to investigate the main prognostic factors and therapeutic management.

\section{METHODS}

We retrospectively analyzed all consecutive patients diagnosed with GC who visited our oncological center, the Veneto Institute of Oncology-IRCCS, in Padua between January 2006 and December 2017. Each patient had to meet the following criteria: age $\geq 18$ years, MRI-T2 or FLAIR sequences showing the interest of at least three cerebral lobes, and with the aspect of diffuse glioma with no contrast enhancement. Histological diagnoses were based on the 2009 or 2016 WHO classification, depending on the year in which the intervention was performed.

Demographic data, age at diagnosis, biopsy execution, histological diagnosis, O6-methylguanin-DNAmethyltransferase (MGMT) promoter methylation, IDH1-2 mutation, type of therapy, radiological aspects, and response and patient outcomes were recorded. In particular, the MGMT promoter methylation status was investigated by polymerase chain reaction (PCR) or pyrosequencing and the IDH1-2 mutation status was performed by immunohistochemistry and PCR; radiological response was retrospectively evaluated by 
an expert neuroradiologist, according to the Response Assessment in Neuro-Oncology (RANO) criteria for diffuse low-grade gliomas ${ }^{[3]}$; patient outcomes were described in terms of progression free survival (PFS) and overall survival (OS).

As regards statistical analysis, PSF was defined as the time between diagnosis and clinical or radiological progression or death, and OS was defined as the time between diagnosis and patient death; both PFS and OS were calculated using Kaplan-Meier survival curves. Univariate analyses were performed for the Eastern Cooperative Oncology Group (ECOG) performance status (PS), MGMT promoter methylation, IDH1 mutation, type of first-line treatment [RT + temozolomide (TMZ), RT alone, or TMZ alone], histological subtype (astrocytoma vs. oligodendroglioma), best response to treatment [stable disease (SD), partial response (PR), complete response (CR), or progressive disease], and second-line treatment (yes $v s$. no).

\section{RESULTS}

After reviewing patient records, 33 patients were eligible for the study. Patient features and tumor characteristics are summarized in Table 1.

Patients included 22 males (67\%) and 11 females (33\%). ECOG PS was $0-1$ in 21 patients (64\%) and $\geq 2$ in 12 patients $(36 \%)$. Biopsy was performed in 22 cases $(67 \%)$; at the histological analysis, $16(73 \%)$ were astrocytomas and $6(27 \%)$ were oligodendrogliomas. MGMT promoter methylation was detected in 14 patients (42\%), and it was methylated in eight (57\%). The IDH1 mutation status was studied in 16 out of 22 cases $(73 \%)$, and it had mutated in 10 patients (63\%). With regard to the type of treatment, 9 patients (27\%) underwent concomitant RT plus TMZ, 22 patients (67\%) only received TMZ, and 2 patients (6\%) received $\mathrm{RT}$ alone; the choice of treatment was at the physician's discretion, essentially based on ECOG PS and extent of disease. We then analyzed the best radiological response on the MRI, finding a complete response in 1 case (3\%), partial response in 9 patients (27\%) and stable disease in 15 patients (45\%). Only eight patients $(25 \%)$ had a progressive disease (Figure 1 shows a partial response in the case of diffuse astrocytoma).

Finally, with regard to patient outcome, we found a PFS of 19.1 months and an OS of 30.7 months among all patients [Figure 2]; according to the ECOG PS, PFS was 34.6 months $v s .3 .4$ months for PS $0-1$ and $\geq 2$ ( $P$ $<0.0001)$, and OS was 42 months vs. 8.9 months $(P<0.0001)$, respectively [Table 2].

With reference to the histological subtype, PFS between astrocytic and oligodendroglial tumors was 41.6 months vs. 28.6 months $(P=0.7)$, while OS was 42.0 months vs. 52.7 months $(P=0.8)$, respectively.

The presence of MGMT methylation was associated with longer PFS (41.6 months $v s .8 .9$ months, $P=0.05$ ) and OS (52.7 months vs. 14.6 months, $P=0.009$ ), while, in regard to $I D H 1$ mutation, PFS was 52.7 months vs. 8.9 months $(P=0.006)$ and OS was 52.7 months vs. 41 months $(P=0.02)$.

It is worth noting that no significant difference was found in relation to the different types of treatment: for the concomitant scheme (RT + TMZ) vs. RT or TMZ alone, PFS was 11.1 months vs. 19.1 months $(P=0.2)$ and OS was 14.7 months $v s .30 .7$ months $(P=0.7)$, respectively.

Moreover, radiological response was correlated with survival; indeed, patients with partial or complete response had a longer OS than those with stable or progressive disease (38.5 months in CR/PR vs. 4.0 months in $\mathrm{PD}, P<0.0001)$. 
Table 1. Patient, tumor, and treatment characteristics

\begin{tabular}{|c|c|}
\hline & $N(\%)$ \\
\hline Patients & 33 \\
\hline \multicolumn{2}{|l|}{ Sex } \\
\hline Male & $22(67)$ \\
\hline Female & $11(33)$ \\
\hline \multicolumn{2}{|l|}{ ECOG PS } \\
\hline $0-1$ & $21(64)$ \\
\hline$\geq 2$ & $12(36)$ \\
\hline \multicolumn{2}{|l|}{ Biopsy } \\
\hline Yes & $22(67)$ \\
\hline No & $11(33)$ \\
\hline \multicolumn{2}{|l|}{ Histology } \\
\hline Astrocytoma & $16(73)$ \\
\hline Oligodendroglioma & $6(27)$ \\
\hline \multicolumn{2}{|l|}{ MGMT status } \\
\hline Methylated & $8 / 14(57)$ \\
\hline Unmethylated & $6 / 14(43)$ \\
\hline \multicolumn{2}{|l|}{ IDH status } \\
\hline Mutated & $10 / 16(63)$ \\
\hline Wild-type & $6 / 16(37)$ \\
\hline \multicolumn{2}{|l|}{ Type of treatment } \\
\hline$R T+T M Z$ & $9(27)$ \\
\hline TMZ alone & $22(67)$ \\
\hline RT alone & $2(6)$ \\
\hline \multicolumn{2}{|l|}{ Radiological response } \\
\hline$C R$ & $1(3)$ \\
\hline PR & $9(27)$ \\
\hline SD & $15(45)$ \\
\hline PD & $8(25)$ \\
\hline Second-line treatment & $13(59)$ \\
\hline$R T+T M Z$ & $1(5)$ \\
\hline CT alone & $10(45)$ \\
\hline RT alone & $2(9)$ \\
\hline
\end{tabular}

ECOG: Eastern Cooperative Oncology Group; PS: performance status; MGMT: O6-methylguanine-DNA-methyltransferase; IDH1: isocitrate dehydrogenase 1; RT: radiation therapy; TMZ: temozolomide; CR: complete response; PR: partial response; SD: stable disease; PD: progressive disease; CT: chemotherapy.

In our cases, 13 of 22 patients underwent second-line therapy after relapsing: of these, 1 patient received RT plus TMZ, while 10 patients received chemotherapy alone (fotemustine in four PT, PCV/PC in four PT, and TMZ in two PT) and 2 patients received RT alone; all of these patients had longer survival, with an OS of 30.7 months compared to 6.5 months for those who did not have second-line treatment $(P=0.04)$.

\section{DISCUSSION}

Gliomatosis cerebri is a rare entity, and it appears very difficult to define better management with multiple studies attempting to investigate it. This work illustrates the real-life management of this disease from a single institution, where the main goal was to deepen possible prognostic factors and treatment significance. 
Table 2. Univariate analysis for progression free survival and overall survival

\begin{tabular}{|c|c|c|c|c|c|c|}
\hline Variable & & & & & & \\
\hline ECOG PS & & & $P<0.0001$ & & & $P<0.0001$ \\
\hline $0-1$ & 34.6 & $8.7-60.6$ & & 42.0 & $27.7-56.3$ & \\
\hline$\geq 2$ & 3.4 & $0.69-6.2$ & & 8.9 & $5.5-12.39$ & \\
\hline MGMT status & & & $P=0.05$ & & & $P=0.009$ \\
\hline Methylated & 41.6 & $8.35-74.9$ & & 52.7 & $40.4-54.6$ & \\
\hline Unmethylated & 8.9 & $1.25-16.5$ & & 14.6 & $7.9-21.4$ & \\
\hline IDH1 status & & & $P=0.006$ & & & $P=0.2$ \\
\hline Mutated & 52.7 & n.a & & 57.7 & n.a & \\
\hline Wild-Type & 8.9 & $0.001-22.9$ & & 41.7 & n.a & \\
\hline First-line treatment & & & $P=0.2$ & & & $P=0.7$ \\
\hline$T M Z+R T$ & 11.1 & $6.7-15.6$ & & 14.7 & $14.5-14.9$ & \\
\hline RT or TMZ alone & 19.1 & $0.00-42.9$ & & 30.7 & $8.7-52.6$ & \\
\hline Histological subtype & & & $P=0.7$ & & & $P=0.8$ \\
\hline Astrocytoma & 41.6 & $10.3-72.9$ & & 42.0 & $24.3-59.6$ & \\
\hline Oligodendroglioma & 28.6 & $9.3-48.0$ & & 52.7 & n.a. & \\
\hline Best response & & & $P<0.0001$ & & & $P<0.0001$ \\
\hline PD & 2.7 & $1.3-4.1$ & & 4.0 & $2.69-14.6$ & \\
\hline SD & 19.1 & 7.3-30.9 & & 30.7 & $23.0-49.1$ & \\
\hline$P R+C R$ & 41.6 & $24.03-59.2$ & & 38.5 & $33.0-59.0$ & \\
\hline Second-line treatment & & & & & & $P=0.04$ \\
\hline Yes & & & & 30.7 & $6.8-54.5$ & \\
\hline No & & & & 6.5 & $0.01-13.8$ & \\
\hline
\end{tabular}

ECOG: Eastern Cooperative Oncology Group; PFS: progression free survival; OS: overall survival; PS: performance status; IDH1: isocitrate dehydrogenase 1; RT: radiation therapy; TMZ: temozolomide; MGMT: O6-methylguanine-DNA-methyltransferase; PD: progressive disease; SD: stable disease; PR: partial response; CR: complete response; n.a.: not available.
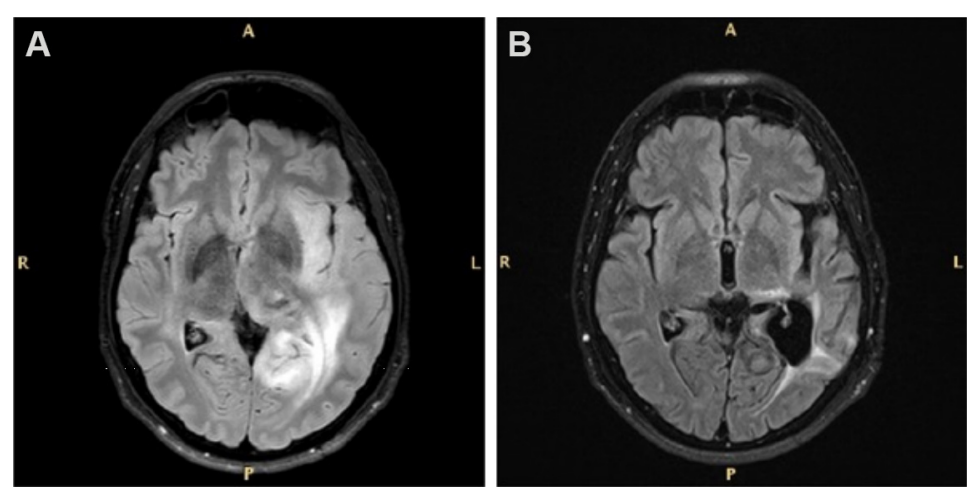

Figure 1. FLAIR magnetic resonance images of a patient with diffuse astrocytoma before (A) and after (B) biopsy, and subsequent temozolomide treatment showing a partial response according to the RANO criteria for low-grade gliomas. FLAIR: Fluid-attenuated inversion recovery; RANO: Response Assessment in Neuro-Oncology.

Based on a recent literature review by Carroll et al. ${ }^{[4]}$, the median OS in GC is between 14.5 and 30 months, with an average of 21 months. Due to its heterogeneous nature, it is still difficult to define clear prognostic factors, although several studies have investigated them: a recent review reports that young age, low-grade histology, good PS, 1p19q codeletion, O6-methylguanin-DNA-methyltransferase (MGMT) promoter methylation, and $I D H 1-R 132 H$-mutation seem to correlate with longer survival ${ }^{[5]}$. In fact, Desestret et al. ${ }^{[6]}$ 

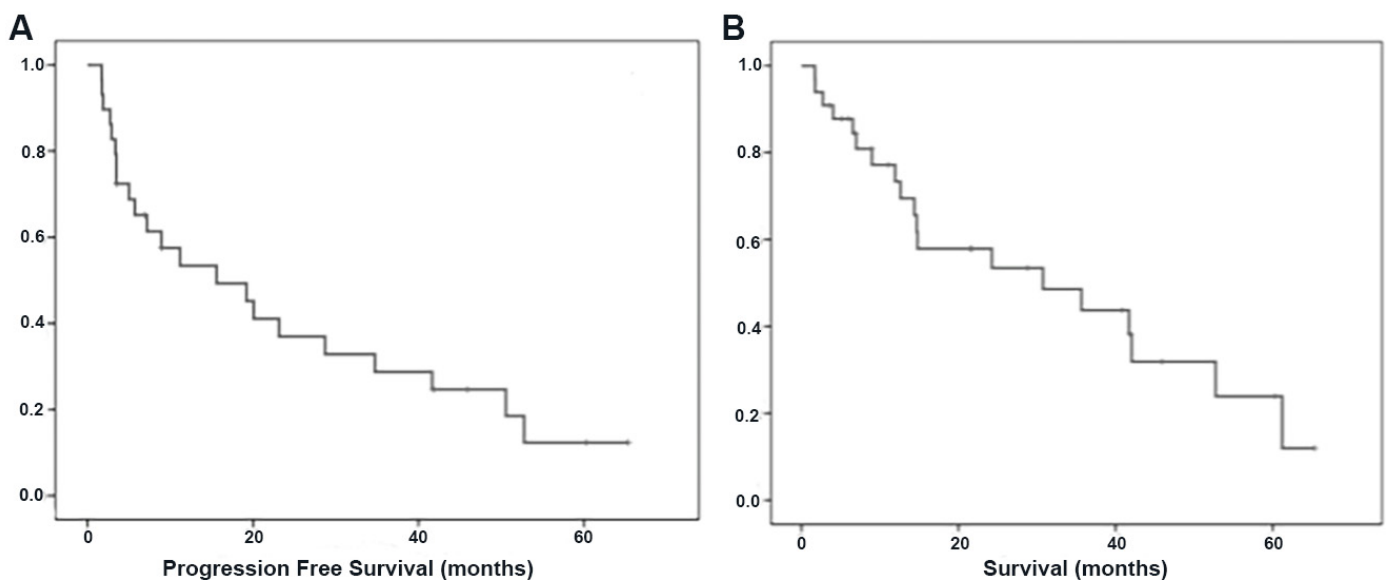

Figure 2. Median progression free survival of 19.1 months (A); and median overall survival of 30.7 months (B).

added that alpha-internexin (INA) expression, in particular when associated with $I D H 1$ mutation, can also be a good prognostic factor.

Because of its infiltrative and diffuse spreading, the surgical approach is limited to biopsy or the minimal debulking of focal areas causing mass effect ${ }^{[7]}$. Radiotherapy (RT) has historically been used in GC, but its effectiveness is still unclear: multiple studies were conducted in this regard, but their results do not agree regarding survival advantage and dose/modalities of treatment; therefore, RT is often used in selected patients suitable to receive it, while weighing the expected benefits with potential neurocognitive side effects $^{[8]}$.

Conversely, chemotherapy (CT) seems to have an important significance in prolonging survival: historically, the regimen procarbazine-lomustine-vincristine (PCV) was the most used treatment, as also supported by the NOA-05 study ${ }^{[9]}$. However, this has been replaced, in most cases, by temozolomide, which has easier administration and better tolerability compared to $\mathrm{PCV}^{[10-12]}$; $\mathrm{CT}$ is usually prescribed as upfront therapy, but it may also be used after RT with a survival benefit ${ }^{[13]}$ or during RT for GC with more aggressive aspects $^{[10]}$.

One limitation in our study is the lack of neurocognitive assessment; this should be an important factor to be analyzed when comparing efficacy results between chemotherapy alone and chemotherapy plus radiotherapy.

In our cases, it appears that only $67 \%$ of patients underwent surgery/biopsy; however, when possible, biopsy should be mandatory to analyze the molecular aspects of GC for choosing the best treatment; indeed, we showed that MGMT methylation can be an important prognostic factor in this tumor, and it is known that patients with MGMT methylation might benefit from temozolomide.

However, in our population, we reported a relatively small number of biopsies, and it could be due to the poor ECOG PS of the patients or the morphological characteristics of the tumor. A recent study analyzing 78 patients showed that a tumor involving the midline brain structure can correlate with a higher perioperative morbidity ${ }^{[14]}$. Hence, the non-surgical approach could also be an appropriate decision. Overall, our opinion is that biopsy should be mandatory for patients in good clinical condition to better define the tumor's nature, prognosis, and the best treatment. 
It could be an interesting perspective for future insights to investigate novel and more specific radiological techniques, such as positron emission tomography (PET-CT) or PET-MRI with amino acid tracers such as ${ }^{18} \mathrm{~F}$-fluoro-ethyl-1-tyrosine (FET) and ${ }^{11} \mathrm{C}$-methyl-L-methionine (MET), which can probably better distinguish more aggressive tumor areas ${ }^{[15]}$.

The reason a similar OS was obtained in patients with oligodendrogliomas and astrocytomas is not clear. Indeed, oligodendrogliomas should have a longer OS than astrocytoma. It is likely that our result could be due to the small number of patients analyzed in this study.

It is also interesting to note that no difference was found between the type of treatment at diagnosis; however, our results could be due to the small population analyzed or the retrospective design of this study. Indeed, the type of treatment was unbalanced among the patients: $67 \%$ of them received chemotherapy alone, as the choice of treatment was at the physician's discretion, which could have influenced clinical outcomes. This is still a much debated topic, with multiple studies published with different results; in most of them, CT and RT seem to have the same results ${ }^{[16,17]}$, so it is reasonable to recommend CT upfront with the aim of limiting neurotoxicity induced by $\mathrm{RT}^{\left[{ }^{[5]}\right.}$. As regards the chemotherapy regime, TMZ resulted safer than PCV with similar results in terms of PFS and $\mathrm{OS}^{[18]}$; however, PCV was the only schedule analyzed in a phase 3 randomized study in patients with anaplastic oligodendroglial tumors. Therefore, it could be correct to use temozolomide in patients with astrocytoma and PCV for an oligodendroglial tumor. However, it appears clear that it is absolutely important to identify predictive factors and focus the therapy on specific targets to obtain a better outcome with minimal toxicity. The recent randomized, phase 3 study (RTOG 9802) analyzing high-risk low-grade glioma patients treated with RT + PCV vs. RT alone demonstrated a longer OS for the combination regimen ${ }^{[19]}$. A subsequent genomic analysis ${ }^{[20]}$ showed that $I D H$-mutant high-risk LGG, regardless of the 1p19q codeletion status, received benefits from the administration of chemotherapy; conversely, in our population, we showed no significant difference between combination or single treatment, although multivariate analysis was not performed due to the small number of patients. In conclusion, based on our results, GC patients with a good performance status, mutated $I D H$, and/or methylated MGMT should be treated with combination therapy, where possible. In the case of advanced disease where RT cannot be performed, CT should be evaluated in selected patients with molecular alterations and second-line therapy could be recommended for patients with a good clinical condition.

\section{DECLARATIONS}

\section{Authors' contributions}

Contributed to patient and data collection: Bellu L, Caccese M, Cerretti C, Berti F, Busato F, Parisi A, Padovan M, Zagonel V, Lombardi G

Wrote the paper: Bellu L, Lombardi G

\section{Availability of data and materials}

All the data are recorded at Veneto Institute of Oncology. No public database exists.

\section{Financial support and sponsorship}

Not applicable.

\section{Conflicts of interest}

All authors declare that there are no conflicts of interest. 


\section{Ethical approval and consent to participate}

The study was approved by the Veneto Institute of Oncology Ethics Commitee.

\section{Consent for publication}

All the patients signed the informed consent form.

\section{Copyright}

(c) The Author(s) 2021.

\section{REFERENCES}

1. Louis DN, Perry A, Reifenberger G, et al. The 2016 World Health Organization Classification of Tumors of the Central Nervous System: a summary. Acta Neuropathol (Berl) 2016;131:803-20. DOI PubMed

2. Taillibert S, Chodkiewicz C, Laigle-Donadey F, Napolitano M, Cartalat-Carel S, Sanson M. Gliomatosis cerebri: a review of 296 cases from the ANOCEF Database and the Literature. J Neurooncol 2006;76:201-5. DOI PubMed

3. van den Bent MJ, Wefel JS, Schiff D, et al. Response assessment in neuro-oncology (a report of the RANO group): assessment of outcome in trials of diffuse low-grade gliomas. Lancet Oncol 2011;12:583-93. DOI PubMed

4. Carroll KT, Hirshman B, Ali MA, et al. Management and survival patterns of patients with gliomatosis cerebri: a SEER-based analysis. World Neurosurg 2017;103:186-93. DOI PubMed

5. Wen PY, Macdonald DR, Reardon DA, et al. Updated response assessment criteria for high-grade gliomas: response assessment in neuro-oncology working group. J Clin Oncol 2010;28:1963-72. DOI PubMed

6. Desestret V, Ciccarino P, Ducray F, et al. Prognostic stratification of gliomatosis cerebri by IDH1R132H and INA expression. $J$ Neurooncol 2011;105:219-24. DOI PubMed

7. Greenfield JP, Castañeda Heredia A, George E, Kieran MW, Morales La Madrid A. Gliomatosis cerebri: a consensus summary report from the First International Gliomatosis cerebri Group Meeting, March 26-27, 2015, Paris, France. Pediatr Blood Cancer 2016;63:2072-7. DOI PubMed

8. Chen S, Tanaka S, Giannini C, et al. Gliomatosis cerebri: clinical characteristics, management, and outcomes. J Neurooncol 2013;112:267-75. DOI PubMed PMC

9. Glas M, Bähr O, Felsberg J, et al. NOA-05 phase 2 trial of procarbazine and lomustine therapy in gliomatosis cerebri. Ann Neurol 2011;70:445-53. DOI PubMed

10. Rudà R, Bertero L, Sanson M. Gliomatosis cerebri: a review. Curr Treat Options Neurol 2014;16:273. DOI PubMed

11. Sanson M, Napolitano M, Cartalat-Carel S, Taillibert S. La gliomatose cérébrale. Rev Neurol (Paris) 2005;161:173-81. DOI PubMed

12. Sanson M, Cartalat-Carel S, Taillibert S, et al. Initial chemotherapy in gliomatosis cerebri. Neurology 2004;63:270. DOI PubMed

13. Kong DS, Kim ST, Lee JI, et al. Impact of adjuvant chemotherapy for gliomatosis cerebri. BMC Cancer 2010;10:424. DOI PubMed PMC

14. Shimony N, Shofty B, Ram Z, Grossman R. Perioperative risk assessment of patients with gliomatosis cerebri. World Neurosurg 2017;98:334-8. DOI PubMed

15. Dunet V, Pomoni A, Hottinger A, Nicod-Lalonde M, Prior JO. Performance of 18F-FET versus 18F-FDG-PET for the diagnosis and grading of brain tumors: systematic review and meta-analysis. Neuro Oncol 2015;18:426-34. DOI PubMed PMC

16. Mattox AK, Lark AL, Adamson DC. Marked response of gliomatosis cerebri to temozolomide and whole brain radiotherapy. Clin Neurol Neurosurg 2012;114:299-306. DOI PubMed

17. Baumert BG, Hegi ME, van den Bent MJ, et al. Temozolomide chemotherapy versus radiotherapy in high-risk low-grade glioma (EORTC 22033-26033): a randomised, open-label, phase 3 intergroup study. Lancet Oncol 2016;17:1521-32. DOI PubMed PMC

18. Wick W, Hartmann C, Engel C, et al. NOA-04 randomized phase III trial of sequential radiochemotherapy of anaplastic glioma with procarbazine, lomustine, and vincristine or temozolomide. J Clin Oncol 2009;27:5874-80. DOI PubMed

19. Buckner JC, Shaw EG, Pugh SL, et al. Radiation plus procarbazine, CCNU, and vincristine in low-grade glioma. $N$ Engl $J$ Med 2016;374:1344-55. DOI PubMed PMC

20. Bell EH, Zhang P, Shaw EG, et al. Comprehensive genomic analysis in NRG oncology/RTOG 9802: a phase III trial of radiation versus radiation plus procarbazine, lomustine (CCNU), and vincristine in high-risk low-grade glioma. J Clin Oncol 2020;38:3407-17. DOI PubMed PMC 